\title{
Regulation of pituitary hormones and cell proliferation by components of the extracellular matrix
}

M. Paez-Pereda ${ }^{1}$,

F. Kuchenbauer ${ }^{1}$,

E. Arzt $^{2}$

and G.K. Stalla ${ }^{1}$

\author{
${ }^{1}$ Max Planck Institute of Psychiatry, Munich, Germany \\ ${ }^{2}$ Laboratorio de Fisiología y Biología Molecular, Departamento de Biología, \\ Facultad de Ciencias Exatas y Naturales, Universidad de Buenos Aires, \\ Buenos Aires, Argentina
}

Correspondence

M. Paez-Pereda

Max Planck Institute of Psychiatry

Kraepelinstr. 10

80804 Munich

Germany

Fax: +49-89-3062-2605

E-mail: marcelo@mpipsykl.mpg.de

Presented at SIMEC 2004

(International Symposium

on Extracellular Matrix),

Angra dos Reis, RJ, Brazil,

September 27-30, 2004.

Received February 16, 2005

Accepted July 28, 2005

$\ldots \ldots \ldots \ldots \ldots \ldots$

\section{Abstract}

The extracellular matrix is a three-dimensional network of proteins, glycosaminoglycans and other macromolecules. It has a structural support function as well as a role in cell adhesion, migration, proliferation, differentiation, and survival. The extracellular matrix conveys signals through membrane receptors called integrins and plays an important role in pituitary physiology and tumorigenesis. There is a differential expression of extracellular matrix components and integrins during the pituitary development in the embryo and during tumorigenesis in the adult. Different extracellular matrix components regulate adrenocorticotropin at the level of the proopiomelanocortin gene transcription. The extracellular matrix also controls the proliferation of adrenocorticotropin-secreting tumor cells. On the other hand, laminin regulates the production of prolactin. Laminin has a dynamic pattern of expression during prolactinoma development with lower levels in the early pituitary hyperplasia and a strong reduction in fully grown prolactinomas. Therefore, the expression of extracellular matrix components plays a role in pituitary tumorigenesis. On the other hand, the remodeling of the extracellular matrix affects pituitary cell proliferation. Matrix metalloproteinase activity is very high in all types of human pituitary adenomas. Matrix metalloproteinase secreted by pituitary cells can release growth factors from the extracellular matrix that, in turn, control pituitary cell proliferation and hormone secretion. In summary, the differential expression of extracellular matrix components, integrins and matrix metalloproteinase contributes to the control of pituitary hormone production and cell proliferation during tumorigenesis.

\section{Introduction}

The extracellular matrix (ECM) is a threedimensional network of proteins, glycosaminoglycans and other macromolecules. It has a structural support function as well as a role in cell adhesion, migration, proliferation, and
Key words

- Integrins

- Pituitary adenomas

- Pituitary hormones

- Cell proliferation
- Extracellular matrix

- Pituitary gland 
cific effects on cell proliferation, differentiation, morphogenesis, and hormone production (1-5). Laminin, for example, is a component of the ECM that determines the histoarchitecture of every organ and provides cells with biological information (6). Laminin is found particularly in basement membranes and in the extracellular matrices that surround epithelial tissues, fat cells, nerves, and muscles (7). It is involved in many physiological processes including cell proliferation, differentiation, motility, and attachment (6,8-10). The effects of the ECM are mediated mainly by surface receptors called integrins, which trigger different cellular responses $(11,12)$. Individual ECM components are able to bind to different integrins (11). Integrins are formed by different $\alpha$ and $\beta$ subunits able to produce more than twenty different integrin combinations (11). During ECM signal transduction, integrins activate multiple downstream effectors in parallel following a complex system of signal integration $(12,13)$. Integrin signaling involves changes in the cell cytoskeleton as well as GTPase and kinase pathways $(1,12,13)$. For example, Rho and Rac, members of the small GTPase family, control the structure of the actin cytoskeleton and cell morphology (14-16). Fibronectin and integrins regulate Rac small GTPase and determine fibroblast morphology. Rac is coupled to the NADPH oxidase complex, which generates reactive oxygen species. An alternative pathway that transduces fibronectin and integrin signals involves Rho, another member of the small GTPase family $(16,17)$. This pathway has been shown to control cell cycle progression in $\mathrm{CHO}$ cells $(3,16)$.

\section{Extracellular matrix effects on the endocrine system}

Relatively little is known about the role of the ECM in the function of the endocrine system in the adult. However, some data show that ECM controls hormone secretion in different glands. ECM proteins regulate testosterone production in Leydig cells (18). In granulosa cells, fibronectin, laminin, collagen I, and collagen IV increase follicle-stimulating hormone receptors and progesterone production (5). Laminin expression has also been detected in various endocrine tissues. In adrenocortical cells, the synthesis of laminin is regulated by adrenocorticotropin hormone (ACTH) (19). Laminin and fibronectin show a differential distribution in the adrenal gland (20). Collagen IV enhances the cortisol secretion in response to ACTH or angiotensin II while fibronectin and laminin decrease the cortisol response $(20,21)$. Thus, the ECM has an important regulatory role in adrenal homeostasis.

\section{The pituitary gland}

The pituitary gland is an interphase between the brain and the rest of the organism. It receives hormonal input from neuroendocrine cells in the hippocampus and secretes hormones that regulate the homeostasis in the whole body. Different hormones are produced in different cell types in the pituitary, such as growth hormone, prolactin, ACTH, follicle-stimulating hormone, and thyroidstimulating hormone. The pituitary also contains folliculo-stellate cells that have a paracrine signaling function and stromal cells. In the pituitary, immunohistochemical studies have shown that laminin is not only expressed in basal membranes but also within endocrine and folliculo-stellate cells (2224). Laminin is suspected to be involved in the early steps of pituitary development (25). It has been shown that laminin, fibronectin and collagen IV are present among the epithelial cells forming the Rathke pouch during pituitary development (25). This suggests that ECM proteins could also be involved in the normal development and differentiation of the pituitary gland. During early postnatal development the pituitary 
undergoes architectural changes that involve migration and ECM remodeling. This process is regulated by epidermal growth factor (26). Furthermore, it has been shown that laminin controls the release of prolactin and gonadotropins in the normal pituitary (27). Collagen IV has also been shown to control the release of prolactin (28).

\section{Extracellular matrix role in tumorigenesis}

ECM components and integrins are not only involved in normal physiological functions but also in tumorigenesis. They can modulate tumor cell proliferation and survival $(9,12,29)$. During cell immortalization, the process of proliferation and survival becomes independent of integrin-mediated cell adhesion to the ECM. This is one of the critical steps of tumor development $(1,12)$. ECM components have been shown to play a role in the development and growth of some types of endocrine tumors. For example, ECM components regulate the proliferative response of mammary epithelial cells to steroid hormones (30). Recent studies connect laminin with tumorigenesis in various endocrine tissues. It has been demonstrated that laminin promotes differentiation in fetal pancreatic cells and human colon adenocarcinoma cells $(31,32)$. Laminin was demonstrated to regulate ovarian cancer cell proliferation as well as thyroid carcinoma cell growth and differentiation $(33,34)$. Laminin is mainly involved in the organization of the basal membrane network and the regulation of cell differentiation in colon cancer cells (32). Laminin was found to regulate the development and growth of breast, prostate and colon cancer $(8,10,35)$. In contrast, in endothelial cells, it has been shown that laminin inhibits cyclin D1 translation, resulting in proliferation arrest (36). This provides a molecular basis for the mechanisms that mediate the proliferative effects of laminin on tumor cells.

\section{Pituitary adenomas}

Pituitary adenomas are composed of adenohypophyseal cells and comprise $15 \%$ of all intracranial tumors. They are quite common, occurring in approximately $10 \%$ of the general population, with equal sex incidence, and with incidence increasing with age. Although they are usually benign, they can give rise to severe clinical syndromes due to the hormonal excess they produce, or to visual/cranial disturbances because of their considerable intracranial mass. Their high clinical importance together with the peculiar biological characteristics they display, on the one hand, and the obscurity that covers the differentiation of such a complex cellular system as the adenohypophysis, on the other, have made pituitary adenomas the center of intensive study during the last decades. However, very few factors are known to be involved in the pathogenesis of pituitary adenomas (37). Most of the factors investigated are expressed similarly in pituitary adenomas and in normal tissue. Important exceptions are the ECM components and integrins, which constitute one of the few clear examples of factors differentially expressed during pituitary pathogenesis.

During the process of pituitary adenoma development fibronectin isoforms are differentially expressed (38). Pituitary adenoma cell transformation correlates with alterations in $\beta 1$-integrin expression (39). $\alpha$-Integrins seem not to be involved in pituitary tumorigenesis except for $\alpha 3$, which is co-expressed with $\beta 1$ and therefore probably transduces signals through 31 -activation (39). In agreement with these results, the proto-oncogene c-fos expression in pituitary adenomas depends on the integrity of the ECM (40). Several laminin isoforms are differentially expressed and distributed in the normal pituitary and pituitary adenomas. Nevertheless, the pathophysiological role of laminin in pituitary tumors is still unclear. Interestingly, in different types of pituitary tumors, lami- 
nin is differentially expressed and localized as compared to the normal pituitary, suggesting a regulatory role for laminin in pituitary tumor development. Taken together, these results suggest a regulatory role for the ECM in pituitary adenoma pathogenesis.

Pituitary adenomas have one particular feature as compared to other tumor types: although they have a high incidence and they are surrounded by highly vascularized tissue, these tumors remain relatively noninvasive and extremely seldom produce metastasis. Most of the pituitary adenomas are considered to be microadenomas, which do not exceed a few millimeters in diameter. Only about $20 \%$ of the pituitary tumors grow as macroadenomas that can compress surrounding tissues. These tumors show invasiveness in only $20-30 \%$ of the cases. The ECM very likely plays a role in the inhibition of invasion and metastasis in these tumors. However, the precise mechanism to explain this paradox remains unknown.

\section{Extracellular matrix effects on ACTH-secreting tumor cells}

ECM components induce functional and morphological changes in ACTH-secreting tumor cells. Laminin, collagen I and collagen IV inhibit ACTH biosynthesis at the level of POMC gene transcription (41). On the other hand, laminin and collagen I inhibit cell proliferation whereas fibronectin and collagen IV have a stimulatory effect (41). These effects probably involve two alternative signal transduction pathways that include the Rho or Rac small GTPases. The differential activation of Rho or Rac and reactive oxygen species production together with the corresponding morphological changes induced by the different ECM components result in different proliferation rates. This model in which the ECM activates different small GTPases and in parallel modifies cell morphology, gene transcription and proliferation is consistent with the models proposed for other cell types (42).
Therefore, these mechanisms could also be extended to hormone-secreting epithelial cells. Fibronectin, laminin and collagen I inhibit $\mathrm{ACTH}$ biosynthesis in the ACTH-secreting tumor cell line AtT-20 (41). However, they have no apparent effects on normal rat pituitary cells. This difference in the regulation of ACTH production could be related to the differences in ECM and integrin expression between normal and adenomatous cells. Moreover, these results, together with the fact that pituitary adenomas contain less laminin than normal pituitary tissue (43), suggest the hypothesis that $\mathrm{ACTH}$-secreting adenomas might produce high levels of ACTH due, in part, to a reduction of laminin-induced inhibition.

Fibronectin and collagen IV significantly stimulate AtT-20 cell proliferation (41). This suggests a role for these ECM components in the progression of pituitary adenomas. Anti-ß1-integrin-activating antibodies also stimulate AtT-20 cell proliferation in agreement with the stimulation produced by fibronectin (41). However, this antibody does not further stimulate proliferation in the presence of fibronectin. This could suggest that fibronectin already produces a maximal stimulation through $B 1-$ integrins. The proliferative action of B1-integrin is consistent with the fact that only this integrin is expressed in most cases of pituitary adenomas (39). Accordingly, it has been shown that paracrine and autocrine factors such as transforming growth factorB1, a cytokine suspected to be involved in pituitary tumorigenesis (44-46), can regulate the expression of individual integrin subunits in other cell types (47). This mechanism could contribute to the change of integrin expression and the consequent change in proliferation in pituitary adenomas.

\section{Extracellular matrix effects on prolactin-secreting tumor cells}

Laminin inhibits the production of prolactin in prolactin-secreting tumor cells. 
However, normal pituitary cells cultured on laminin substrates do not show this inhibition. These differential effects could reflect a change in integrin expression during pituitary tumor development (39). These results suggest a possible regulatory role of laminin in prolactin secretion in tumor cells.

Besides affecting prolactin production, laminin also inhibits prolactinoma cell proliferation, suggesting a role for laminin in tumor progression. In fact, differential expression of laminin has been observed during tumor formation in a mouse model of prolactinoma. $\mathrm{D} 2 \mathrm{R}^{-/-}$mice are characterized by the spontaneous development of pituitary hyperplasia and the later formation of prolactinomas $(48,49)$. Laminin expression is very high in the normal pituitaries taken from wild-type mice as well as in the normal pituitaries from $\mathrm{D} 2 \mathrm{R}^{-/-}$animals before hyperplasia or tumor development (50). In the normal pituitary, laminin is distributed in the basal membrane around blood vessels and in the parenchyma surrounding most of the cells. Therefore, in the normal pituitary, most of the cells are in contact with a laminincontaining substrate. However, in hyperplastic pituitary tissue from $\mathrm{D} 2 \mathrm{R}^{-/-}$mice, a strong reduction of laminin expression is observed, mainly in the intercellular parenchyma (50). Furthermore, in fully grown prolactinomas from $\mathrm{D} 2 \mathrm{R}^{-/-}$mice, the amount of laminin expression is dramatically reduced and is restricted to the basal membrane surrounding blood vessels (50). Therefore, starting from early stages of prolactinoma development, pituitary cells are less exposed to laminin as compared to the normal pituitary tissue and this situation is even more evident in prolactinomas. This reduction in laminin expression and the restriction of laminin localization along the sequential steps of prolactinoma development also occur in human prolactinomas at different stages of development. Since laminin inhibits prolactinoma cell proliferation, the fact that prolactinomas contain less laminin than normal pituitary tissues could create a favorable environment for prolactin-secreting cells to proliferate faster and increase hormone secretion. Changes in the ECM already occur at an early stage of pituitary tumor development and may produce pathophysiological effects on the growth of pituitary tumors. The reduction of laminin in prolactinomas could represent a more general reduction of the basement membrane with the consequent reduction in the presence of other ECM components such as collagen IV. Therefore, other ECM components could also play an important role in the development of prolactinomas.

\section{Extracellular matrix remodeling in pituitary adenomas}

Not only the expression of different ECM components is involved in pituitary tumorigenesis but also the remodeling of the existing ECM. The ECM can be degraded and reorganized by specific enzymes from the family of matrix metalloproteinase (MMP). These enzymes are usually associated with tumor invasion and metastasis. Surprisingly, there is an exceptionally high expression of MMPs and low levels of their endogenous inhibitor, TIMP-1, in human pituitary adenomas in contrast to the fact that these tumors are benign and very rarely metastatic (51). In the normal pituitary, high levels of MMP expression have also been detected but in this case, only the inactive forms of the enzymes (pro-MMPs) have been found. Although the active forms of the MMPs have not been found in the normal pituitary, local activation in restricted areas or under specific physiological conditions might allow MMPs to control growth and hormone secretion in normal cells. The expression of pro-MMPs in normal pituitaries further confirms the putative role of MMPs in other processes different than invasion, such as regulation of growth and hormone secretion. The active form of MMP-2 is only found in 
some pituitary tumors (51). The pharmacological inhibition of MMP activity with batimastat results in an inhibition of the proliferation and hormone secretion of pituitary tumor cells (51). This suggests that ECM remodeling is necessary to release growth factors from the ECM. This is further supported by our observation that after ECM digestion with collagenase and hyaluronidase, c-fos expression is abolished in pituitary tumor cells (40). Although other mechanisms could be involved, and further studies are necessary to confirm this hypothesis, we speculate that the release of growth factors from the ECM would be necessary to keep c-fos expression and hence cell proliferation. These factors could be cryptic fragments of ECM components that can be released after proteolysis and have been found to regulate cell proliferation such as laminin fragments or epidermal growth factor-like domains. Alternatively, the MMP could release conventional growth factors that are only anchored to the ECM.

Although MMPs are usually associated with tumor invasiveness, they seem to play a role in early tumor cell proliferation in the pituitary. This interpretation is in agreement with a previously proposed role for MMPs in early tumor development and growth $(52,53)$. It was shown that matrilysin (MMP7) is necessary for the growth of early adenomatous foci in colon cancer. In matrilysin null mutants the early stages of colon tumor growth seem to require MMP-2. Therefore, it was proposed that MMP-2 could be an alternative pathway for the action of matrilysin in tumor growth (54). The MMP-2 expression in pituitary adenomas agrees with this view and further supports a role in early tumor development for MMP-2 that, unlike matrilysin, is more often expressed in aggressive cancer. The participation of MMPs in the proliferation of pituitary cells could provide the basis to understand the mechanisms of early development of other tumor types and suggests that therapies based on MMP inhibition could be applied not only to metastatic but also to benign tumors. The high expression of MMP-2 and MMP-9 in pituitary adenomas and their involvement in cell proliferation and hormone secretion makes these two enzymes good candidates for therapeutic intervention with pharmaceutical inhibitors.

\section{Concluding remarks}

There is compelling evidence for the association between pituitary tumor development and changes in the expression of ECM components, integrins and MMP. The differential expression of ECM components and MMP is one of the few consistent findings in pituitary adenomas, which are otherwise very similar to normal tissue. Therefore, a more detailed examination of the different ECM components as well as integrin subunits and ECM-degrading enzymes is necessary to better understand the formation and progression of pituitary tumors. One of the questions that remain open is why pituitary tumors do not normally progress to malignant carcinomas despite their high incidence, high MMP activity and vascularization. Understanding the role of the ECM in the development of these tumors could potentially be a key to our better understanding and control of the mechanisms that contribute to malignant tumor transformation.

\section{References}

1. Lukashev M \& Werb Z (1998). ECM signaling: orchestrating cell behavior and misbehavior. Trends in Cell Biology, 8: 437-441.

2. Lin CQ \& Bissell MJ (1993). Multi-faceted regulation of cell differentiation by extracellular matrix. FASEB Journal, 7: 737-743.
3. Bourdoulous S, Orend G, MacKenna DA et al. (1998). Fibronectin matrix regulates activation of Rho and CDC42 GTPases and cell cycle progression. Journal of Cell Biology, 143: 267-276.

4. Hay E (1993). Extracellular matrix alters epithelial differentiation. 
Current Opinion in Cell Biology, 5: 1029-1035.

5. Sites CK, Kessel B \& LaBarbera AR (1996). Adhesion proteins increase cellular attachment, follicle-stimulating hormone receptors, and progesterone production in cultured porcine granulosa cells. Proceedings of the Society for Experimental Biology and Medicine, 212: 78-83.

6. Aumailley M \& Gayraud B (1998). Structure and biological activity of the extracellular matrix. Journal of Molecular Medicine, 76: 253-265.

7. Martin GR \& Timpl R (1987). Laminin and other basement membrane components. Annual Review of Cell Biology, 3: 57-85.

8. Giannelli G \& Antonaci S (2000). Biological and clinical relevance of laminin-5 in cancer. Clinical and Experimental Metastasis, 18: 439443.

9. Varner JA \& Cheresh DA (1996). Integrins and cancer. Current Opinion in Cell Biology, 8: 724-730.

10. Kitayama J, Nagawa H, Tsuno $\mathrm{N}$ et al. (1999) Laminin mediates tethering and spreading of colon cancer cells in physiological shear flow. British Journal of Cancer, 80: 1927-1934.

11. Hynes RO (1992). Integrins: versatility, modulation, and signaling. Cell, 69: 11-25.

12. Giancotti FG \& Ruoslahti E (1999). Integrin signaling. Science, 285: 1028-1032.

13. Giancotti FG (2000). Complexity and specifity of integrin signalling. Nature Cell Biology, 2: E13-E14.

14. Schoenwaelder SM \& Burridge K (1999). Bidirectional signaling between the cytoskeleton and integrins. Current Opinion in Cell Biology, 11: 274-286.

15. Nobes CD \& Hall A (1995). Rho, Rac, and Cdc 42 GTPases regulate the assembly of multimolecular focal complexes associated with actin stress fibers, lamellipodia, and filopodia. Cell, 81: 53-62.

16. Hall A (1998). Rho GTPases and the actin cytoskeleton. Science, 279: 509-513.

17. Cussac D, Leblanc P, L'Heritier A et al. (1996). Rho proteins are localized with different membrane compartments involved in vesicular trafficking in anterior pituitary cells. Molecular and Cellular Endocrinology, 119: 195-206.

18. Diaz ES, Pellizzari E, Meroni S et al. (2002). Effect of extracellular matrix proteins on in vitro testosterone production by rat Leydig cells. Molecular Reproduction and Development, 61: 493-503.

19. Pellerin S, Keramidas M, Chambaz EM et al. (1997). Expression of laminin and its possible role in adrenal cortex homeostasis. Endocrinology, 138: 1321-1327.

20. Chamoux E, Narcy A, Lehoux JG et al. (2002). Fibronectin, laminin, and collagen IV as modulators of cell behavior during adrenal gland development in the human fetus. Journal of Clinical Endocrinology and Metabolism, 87: 1819-1828.

21. Chamoux E, Narcy A, Lehoux JG et al. (2002). Fibronectin, laminin, and collagen IV interact with ACTH and angiotensin II to dictate specific cell behavior and secretion in human fetal adrenal cells in culture. Endocrine Research, 28: 637-640.

22. Tougard C, Louvard D, Picart R et al. (1985). Immunocytochemical localization of laminin in rat anterior pituitary cells in vivo and in vitro. In Vitro Cellular and Developmental Biology, 21: 57-61.

23. Vila-Porcile E, Picart R, Tixier-Vidal A et al. (1987). Cellular and subcellular distribution of laminin in adult rat anterior pituitary. Journal of Histochemistry and Cytochemistry, 35: 287-299.

24. de Carvalho DF, Silva KL, de Oliveira DA et al. (2000). Characterization and distribution of extracellular matrix components and receptors in GH3B6 prolactin cells. Biology of the Cell, 92: 351-362.

25. Horacek MJ, Thompson JC, Dada MO et al. (1993). The extracellular matrix components laminin, fibronectin, and collagen IV are present among the epithelial cells forming Rathke's pouch. Acta Anatomica, 147: 69-74.

26. Gonzalez B, Solano-Agama Mdel C, Gonzalez Del Pliego M et al. (2004). Differences in cell migration of cultured pituitary cells from infantile and adult rats: participation of the extracellular matrix and epidermal growth factor. International Journal of Developmental Neuroscience, 22: 231-239.

27. Denduchis B, Rettori V \& McCann S (1994). Role of laminin on prolactin and gonadotrophin release from anterior pituitaries of male rats. Life Sciences, 55: 1757-1765.

28. Diaz ES, Rettori V, Suescun MO et al. (2002). Role of type IV collagen in prolactin release from anterior pituitaries of male rats. Endocrine, 18: 185-189.

29. Sethi T, Rintoul RC, Moore $S$ et al. (1999). Extracellular matrix proteins protect small cell lung cancer cells against apoptosis: a mechanism for small cell lung cancer growth and drug resistance in vivo. Nature Medicine, 5: 662-668.

30. Xie J \& Haslam SF (1997). Extracellular matrix regulates ovarian hormone-dependent proliferation of mouse mammary epithelial cells. Endocrinology, 138: 2466-2473.

31. Jiang FX, Cram DS, DeAizpurua HJ et al. (1999). Laminin-1 promotes differentiation of fetal mouse pancreatic beta cells. Diabetes, 48: 722-730.

32. De Arcangelis A, Neuville P, Boukamel R et al. (1996). Inhibition of laminin alpha 1-chain expression leads to alteration of basement membrane assembly and cell differentiation. Journal of Cell Biology, 133: 417-430.

33. Yoshida Y, Hosokawa K, Dantes A et al. (2001). Role of laminin in ovarian cancer tumor growth and metastasis via regulation of Mdm2 and Bcl-2 expression. International Journal of Oncology, 18: 913921.

34. Lekmine F, Lausson S, Pidoux E et al. (1999). Influence of laminin substratum on cell proliferation and CALC I gene expression in medullary thyroid carcinoma C cell lines. Molecular and Cellular Endocrinology, 157: 181-189.

35. De lorio P, Midulla C, Pisani T et al. (2001). Implication of laminin and collagen type IV expression in the progression of breast carcinoma. Anticancer Research, 21: 1395-1399.

36. Mettouchi A, Klein S, Guo W et al. (2001). Integrin-specific activation of Rac controls progression through the $\mathrm{G}(1)$ phase of the cell cycle. Molecular Cell, 8: 115-127.

37. Paez-Pereda M, Giacomini D, Refojo D et al. (2003). Involvement of bone morphogenetic protein 4 (BMP-4) in pituitary prolactinoma pathogenesis through a Smad/estrogen receptor crosstalk. Proceedings of the National Academy of Sciences, USA, 100: 10341039.

38. Farnoud MR, Farhadian F, Samuel JL et al. (1995). Fibronectin isoforms are differentially expressed in normal and adenomatous human anterior pituitaries. International Journal of Cancer, 61: 2734.

39. Farnoud MR, Veirana N, Samuel JL et al. (1996). Adenomatous transformation of the human anterior pituitary is associated with alterations in integrin expression. International Journal of Cancer, 67: 45-53.

40. Páez Pereda M, Goldberg V, Chervin A et al. (1996). Interleukin-2 (IL-2) and IL-6 regulate c-fos proto-oncogene expression in human pituitary adenoma explants. Molecular and Cellular Endocrinology, 124: 33-42.

41. Kuchenbauer F, Hopfner U, Stalla J et al. (2001). Extracellular matrix components regulate ACTH production and proliferation in corticotroph tumor cells. Molecular and Cellular Endocrinology, 175: 
141-148.

42. Chen CS, Mrksich M, Huang S et al. (1997). Geometric control of cell life and death. Science, 276: 1425-1428.

43. Farnoud MR, Lissak B, Kujas M et al. (1992). Specific alterations of the basement membrane and stroma antigens in human pituitary tumours in comparison with the normal anterior pituitary. An immunocytochemical study. Virchows Archiv. A, Pathological Anatomy and Histopathology, 421: 449-455.

44. Asa SL \& Ezzat S (1998). The cytogenesis and pathogenesis of pituitary adenomas. Endocrine Reviews, 19: 798-827.

45. Ray D \& Melmed S (1997). Pituitary cytokine and growth factor expression and action. Endocrine Reviews, 18: 206-228.

46. Arzt E, Pereda MP, Castro CP et al. (1999). Pathophysiological role of the cytokine network in the anterior pituitary gland. Frontiers in Neuroendocrinology, 20: 71-95.

47. Heino J, Ignotz RA, Hemler ME et al. (1989). Regulation of cell adhesion receptors by transforming growth factor-b. Journal of Biological Chemistry, 264: 380-388.

48. Kelly MA, Rubinstein M, Asa SL et al. (1997). Pituitary lactotroph hyperplasia and chronic hyperprolactinemia in dopamine D2 receptor-deficient mice. Neuron, 19: 103-113.
49. Asa SL, Kelly MA, Grandy DK et al. (1999). Pituitary lactotroph adenomas develop after prolonged lactotroph hyperplasia in dopamine D2 receptor-deficient mice. Endocrinology, 140: 5348-5355.

50. Kuchenbauer F, Theodoropoulou M, Hopfner U et al. (2003). Laminin inhibits lactotroph proliferation and is reduced in early prolactinoma development. Molecular and Cellular Endocrinology, 207: 1320.

51. Páez Pereda M, Ledda MF, Goldberg V et al. (1999). High levels of metalloproteinase regulate proliferation and hormone secretion in pituitary cells. Journal of Clinical Endocrinology and Metabolism, 85: 263-269.

52. Chambers A \& Matrisian LM (1997). Changing views of the role of matrix metalloproteinase in metastasis. Journal of the National Cancer Institute, 89: 1260-1270.

53. Werb Z (1997). ECM and cell surface proteolysis: regulating the cellular ecology. Cell, 91: 439-442.

54. Wilson CL, Heppner KJ, Labosky PA et al. (1997). Intestinal tumorigenesis is suppressed in mice lacking the metalloproteinase matrilysin. Proceedings of the National Academy of Sciences, USA, 94: 1402-1407. 